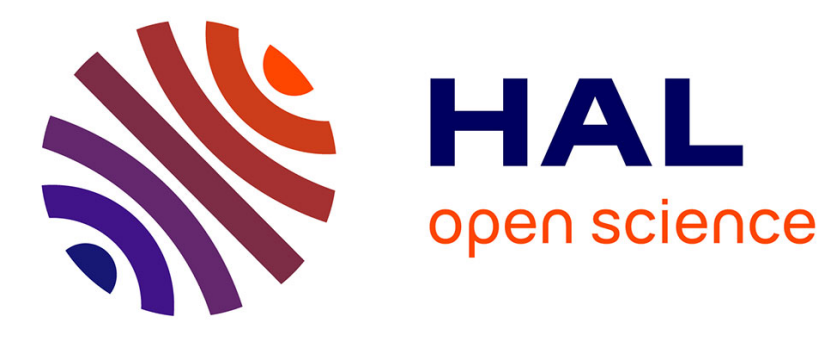

\title{
Bandwidth Estimation for IEEE 802.11-based Ad Hoc Networks
}

Cheikh Sarr, Claude Chaudet, Guillaume Chelius, Isabelle Guérin-Lassous

\section{To cite this version:}

Cheikh Sarr, Claude Chaudet, Guillaume Chelius, Isabelle Guérin-Lassous. Bandwidth Estimation for IEEE 802.11-based Ad Hoc Networks. IEEE Transactions on Mobile Computing, 2008, 7 (10), pp.1228-1241. 10.1109/TMC.2008.41 . inria-00384832

\section{HAL Id: inria-00384832 \\ https://hal.inria.fr/inria-00384832}

Submitted on 15 May 2009

HAL is a multi-disciplinary open access archive for the deposit and dissemination of scientific research documents, whether they are published or not. The documents may come from teaching and research institutions in France or abroad, or from public or private research centers.
L'archive ouverte pluridisciplinaire HAL, est destinée au dépôt et à la diffusion de documents scientifiques de niveau recherche, publiés ou non, émanant des établissements d'enseignement et de recherche français ou étrangers, des laboratoires publics ou privés. 


\title{
Bandwidth Estimation for IEEE 802.11-Based Ad Hoc Networks
}

\author{
Cheikh Sarr, Claude Chaudet, Guillaume Chelius, and Isabelle Guérin Lassous
}

\begin{abstract}
Since 2005, IEEE 802.11-based networks have been able to provide a certain level of quality of service (QoS) by the means of service differentiation, due to the IEEE 802.11e amendment. However, no mechanism or method has been standardized to accurately evaluate the amount of resources remaining on a given channel. Such an evaluation would, however, be a good asset for bandwidth-constrained applications. In multihop ad hoc networks, such evaluation becomes even more difficult. Consequently, despite the various contributions around this research topic, the estimation of the available bandwidth still represents one of the main issues in this field. In this paper, we propose an improved mechanism to estimate the available bandwidth in IEEE 802.11-based ad hoc networks. Through simulations, we compare the accuracy of the estimation we propose to the estimation performed by other state-of-the-art QoS protocols, BRuIT, AAC, and QoS-AODV.
\end{abstract}

Index Terms-Wireless communications, IEEE 802.11, ad hoc networks, quality of service, available bandwidth estimation.

\section{INTRODUCTION}

A D hoc networks are autonomous, self-organized, wireless, and mobile networks. They do not require setting up any fixed infrastructure such as access points, as the nodes organize themselves automatically to transfer data packets and manage topology changes due to mobility. Many of the current contributions in the ad hoc networking community assume that the underlying wireless technology is the IEEE 802.11 standard due to the broad availability of interface cards and simulation models. This standard provides an ad hoc mode, allowing mobiles to communicate directly. As the communication range is limited by regulations, a distributed routing protocol is required to allow long distance communications. However, this standard has not been targeted especially for multihop ad hoc operation, and it is therefore not perfectly suited to this type of networks.

Nowadays, several applications generate multimedia data flows or rely on the proper and efficient transmission of sensitive control traffic. These applications may benefit from a quality of service (QoS) support in the network. That is why this domain has been extensively studied and more and more QoS solutions are proposed for ad hoc networks. However, the term QoS is vague and gathers several concepts. Some protocols intend to offer strong guarantees

- C. Sarr is with the Ecole Polytechnique de Thiès, Université de Thiès BP A 10, Thiès, Senegal.E-mail: Cheikh.Sarr@insa-lyon.fr.

- C. Chaudet is with the Institut Telecom, Telecom ParisTech, LTCI CNRS UMR 5141, 37/39 rue Dareau, 75014 Paris, France. E-mail: Claude.Chaudet@enst.fr.

- G. Chelius is with INRIA ARES, Laboratoire CITI, INSA de Lyon, Batiment Leonard de Vinci, 21 avenue Jean Capelle, 69621 Villeurbanne, France. E-mail: guillaume.chelius@inria.fr.

- I.G. Lassous is with LIP, ENS Lyon, Université Lyon 1, 46 allée d'Italie, 69364 Lyon Cedex 07, France.

E-mail: Isabelle.Guerin-Lassous@ens-lyon.fr.

Manuscript received 30 Mar. 2007; revised 21 Dec. 2007; accepted 20 Feb. 2008; published online 6 Mar. 2008.

For information on obtaining reprints of this article, please send e-mail to tmc@computer.org, and reference IEEECS Log Number TMC-2007-03-0089. Digital Object Identifier no. 10.1109/TMC.2008.41. to the applications on the transmission characteristics, for instance bandwidth, delay, packet loss, or network load. Other solutions, which seem more suited to a mobile environment, only select the best route among all possible choices regarding the same criteria. In both cases, an accurate evaluation of the capabilities of the routes is necessary. Most of the current QoS proposals leave this problem aside, relying on the assumption that the link layer protocols are able to perform such an evaluation. However, they are not. The resource evaluation problem is far from being trivial as it must take into account several phenomena related to the wireless environment but also dependent on less measurable parameters such as the node mobility.

Throughout this paper, we will focus on one of the fundamental resources: throughput. Estimating the remaining bandwidth at a given time and in a given part of the network is tricky because, in a wireless network, the medium is shared between close nodes. Consequently, computing the available bandwidth between two neighbor nodes necessitates an accurate identification of all potential contenders at the emitter's side, of all potential scramblers at the receiver's side, and a proper evaluation of their impact. Information about nodes' utilization of the shared resource should, therefore, be gathered and composed to derive the amount of free resources. Both tasks are usually difficult to realize and they become even harder in sparse networks, as two nodes may share the medium without being able to directly exchange information.

In this paper, we present a new method to evaluate the available bandwidth in ad hoc networks based on the IEEE 802.11 MAC layer. This method uses the nodes' carrier sense capability combined to other techniques such as collision prediction to perform this estimation. It provides upper layers with an evaluation that represents an acceptable compromise between accuracy and measurement cost. Finally, even though it is closely linked to a particular technology, it may easily be adapted to similar random medium access protocols.

In wireless ad hoc networks, a flow could easily take over the whole capacity of the network due to the strong 
interdependency between wireless links. Therefore, it is necessary to take into account the disruption that may be introduced in the network by the addition of a new flow and to distinguish between the raw throughput that may be transferred along a path and the maximum data rate that may be transferred without any noticeable interference.

Hereafter, we define the available bandwidth between two neighbor nodes as the maximum throughput that can be transmitted between these two peers without disrupting any already ongoing flow in the network. We will denote by link capacity the maximum throughput a flow can achieve between two neighbor nodes regardless of other flows present in the network.

As much literature on this topic is now available, we will consider in the remaining of this paper that the reader knows the IEEE 802.11 standard's DCF mode. The rest of this paper is organized as follows: Section 2 presents related works. Section 3 introduces the general principles of available bandwidth estimation (ABE) and Section 4 describes the integration of $\mathrm{ABE}$ into AODV. For performance evaluation, $A B E$ has been integrated into the routing protocol AODV. Finally, NS-2 simulation results are presented in Section 5.

\section{Related Work}

Available bandwidth evaluation has generated several contributions in the wired and wireless networking communities. Several classifications of these solutions may be imagined. We chose to separate them into the following two categories:

- We designate by active approaches the techniques that rely on the emission of dedicated end-to-end probe packets to estimate the available bandwidth along a path.

- We designate by passive approaches the techniques that use only local information on the utilization of the bandwidth. A typical example of such approaches is a node monitoring the channel usage by sensing the radio medium. These mechanisms are usually transparent, but they may exchange information via one-hop broadcasts, as such information can be piggybacked in the Hello messages used by many routing protocols to discover the local topology.

\subsection{Active Bandwidth Estimation Techniques}

A detailed survey of the different techniques to evaluate the available bandwidth in wired networks is accessible in [1]. Most of these techniques measure the end-to-end available bandwidth by sending packets of equal size from a source to a receiver. The source increases gradually the probe packet emission rate. Measurements of the characteristics of this particular flow are performed at the receiver's side and then converted into an estimation of the end-to-end available bandwidth. Several protocols such as SLoPS [2] or TOPP [3] fall into this category. They mainly differ in the way they increase the packet sequence rate and in the metrics measured on the probing packet flow. It is worth noting that, with these techniques, the probing traffic may influence existing flows.

Li et al. [4] propose to detect the presence of congestion by monitoring probe packets' delay. Whenever this delay gets larger than the theoretical maximum delay, the medium suffers from congestion. They propose a method to compute the medium utilization from such measurements and then derive the channel capacity from this channel usage ratio.

Based on the TOPP method, the authors of DietTOPP [5] evaluate the accuracy of such techniques in wireless networks. This paper shows that both the probe packet size and the volume of cross-traffic have a stronger impact on the measured bandwidth in this environment than in wired networks. These techniques are, therefore, also very sensitive to the measurement parameters and easily lead to inaccurate results in a wireless environment.

The active techniques cited above present, in addition, two major drawbacks regarding multihop ad hoc networks. First, when many nodes need to perform such an evaluation for several destinations, the amount of probe packets introduced in the network becomes important. It may, thus, interact with the data traffic and with other probes, modifying other estimations. Second, an end-to-end evaluation technique may not be as reactive as a local technique in a mobile context. When updating routes in response to node mobility or to a change in the available resources, local detection and reconstruction may be more efficient in several situations.

\subsection{Passive Bandwidth Estimation Techniques}

A dynamic bandwidth management scheme for single-hop ad hoc networks is proposed in [6]. In this solution, one node in the network hosts the Bandwidth Manager process, which is responsible for evaluating the available bandwidth in the cell and for allocating the bandwidth to each peer. Each node may ask the Bandwidth Manager for an exclusive access to the channel during a proportion of time using dedicated control messages. As the topology is reduced to a single cell, the available proportion time-share is computed by this entity considering that the total load is the sum of the individual loads. The available fraction of time may then be translated into an available bandwidth by considering the capacity of the wireless link, called total bandwidth in this paper, which is deduced from a measurement of the data packets' throughput. This approach can be considered as passive as very few control packets are exchanged, usually of small size. However, this solution is adapted to network topologies where all the nodes are within communication range but cannot be directly used in multihop ad hoc networks.

Even if the election, the synchronization, and the maintenance of several Bandwidth Managers may represent a significant cost in large distributed networks, similar measurements may be employed. When a node desires to estimate the bandwidth available in its vicinity, the intuitive approach consists in monitoring the channel over a given time period and to deduce from this observation the utilization ratio of the shared resource. The method proposed in [7] uses such technique and adds a smoothing factor to hide transient effects. The QoS routing protocol 
designed in this paper is based on a simple estimation of the available bandwidth by each node and does not consider any interfering nodes.

QoS-AODV [8] also performs such a per-node ABE. The evaluation mechanism constantly updates a value called Bandwidth Efficiency Ratio (BWER), which is the ratio between the numbers of transmitted and received packets. The available bandwidth is simply obtained by multiplying the BWER value by the channel capacity. This ratio is broadcasted among the one-hop neighbors of each node through Hello messages. The bandwidth available to a node is then inferred from these values as the minimum of the available bandwidths over a closed single-hop neighborhood. QoS-AODV, therefore, considers not only the possibility to send a given amount of data but also the effect of the emissions of a node on its neighborhood.

In [9], Chaudet and Lassous proposed a bandwidth reservation protocol called Bandwidth Reservation under InTerferences influence (BRuIT). This protocol's ABE mechanism takes into account the fact that, with the IEEE 802.11 standard, the carrier sense radius is larger than the transmission range. In other words, emitters share the bandwidth with other nodes they cannot communicate with. Experimental studies have shown that this carrier sense radius is at least twice the communication radius. To address this issue, each node regularly broadcasts to all its immediate neighbors information about the total bandwidth it uses to route and emit flows (deduced from applications and routing information) and its estimated available bandwidth. It also transmits similar information concerning all its one-hop neighbors, propagating such information at a two-hop distance. Each node then performs admission control based on this two-hop neighborhood knowledge. When the carrier sense radius is equal to twice the communication radius, the authors have shown that two-hop communication represents the best compromise between estimation accuracy and cost [10].

Making the same observation, Yaling and Kravets [11] proposed the Contention Aware Admission Control Protocol (CACP). In this framework, each node first computes its local proportion of idle channel time by monitoring the radio medium. Then, the authors propose three different techniques to propagate this information to the greatest number of nodes within the carrier sense area. First, similarly to BRuIT, they propose to include the information in Hello messages to reach the two-hop neighborhood. Second, they propose to increase the nodes' transmission power; however, this emission power is often limited by regulations and this technique may therefore only be applicable when power control is used for regular transmissions. Finally, receiving nodes can also reduce their sensitivity in order to decode information coming from farther away, which depends on the quality of electronics and on the signal modulation. Similarly to [12], the authors also point out the existence of intraflow contention. When a flow takes a multihop route, successive routers contend for channel access for frames belonging to the same flow. It is thus important to take into account at least the route length when performing admission control. Ideally, the exact

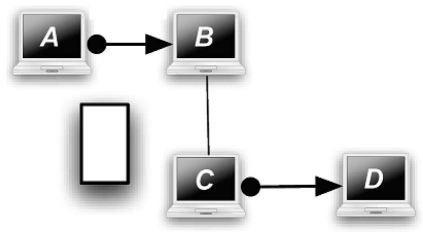

Fig. 1. A typical unfair scenario in which asymmetric conditions degrade sender-based evaluations.

interactions between nodes along a path should be identified and considered.

Finally, the AAC protocol, proposed in [13], makes each node consider the set of potential contenders as a single node. It measures the activity period durations and considers that any such period can be seen as a frame emission of the corresponding length. With this mechanism, collisions and distant emissions are also considered when computing the medium occupancy. Based on this measurement, each node is able to evaluate its available bandwidth. It exchanges this information with its neighbors to compute the bandwidth on each link, a link being defined as a pair of nodes. This value is defined as the minimum between the available bandwidths of both ends. AAC also takes into account the intraflow contention problem mentioned above.

\subsection{Motivation}

We already mentioned that the active techniques presented above do not yield to accurate results in a wireless ad hoc context. They do not consider the need for preserving existing flow service level when computing the available bandwidth. They also introduce additional traffic in the network that may disturb the network operation, and simultaneous measurements may interfere. Finally, in a mobile context, they require frequent reevaluations of the available bandwidths and, therefore, do not facilitate local route reconstruction. The previously described passive techniques also lead, as further simulation results will show, to an inaccurate estimation. Indeed, they all tackle partially the problem, often reducing the evaluation to the sender's side of the links. Their accuracy could be improved, for example, by considering the synchronization or lack of synchronization of parallel emitters. If parallel emitters are badly synchronized, repetitive collisions can happen on a link. To illustrate this phenomenon, let us consider the scenario depicted in Fig. 1. This configuration, initially presented in [14], is a well-known unfair scenario.

Let us consider that a constant bit rate flow is present on link $(C, D)$. We would like to compute the available bandwidth on link $(A, B)$ as a function of the $(C, D)$ flow throughput. In this situation, the evaluations performed by BRuIT, CACP, and AAC are all identical and their value is represented in Figs. $2 \mathrm{a}$ and $2 \mathrm{~b}$ by the "estimated available bandwidth" curve. This graph also presents the simulation results obtained by using the NS-2 simulator with 2- and 11-megabits per second (Mbps) medium capacity, corresponding, respectively, to a 1.6- and 5-Mbps applicationlayer achievable throughput. For all these protocols, the available bandwidth on link (A, B) corresponds to the available bandwidth value computed by node $B$, which is equal to the value computed by node $C$. It is equal to the 


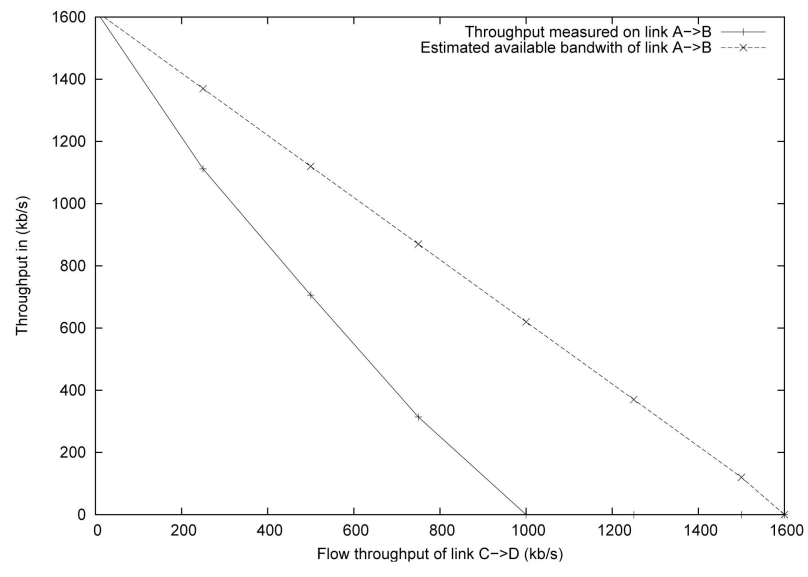

(a)

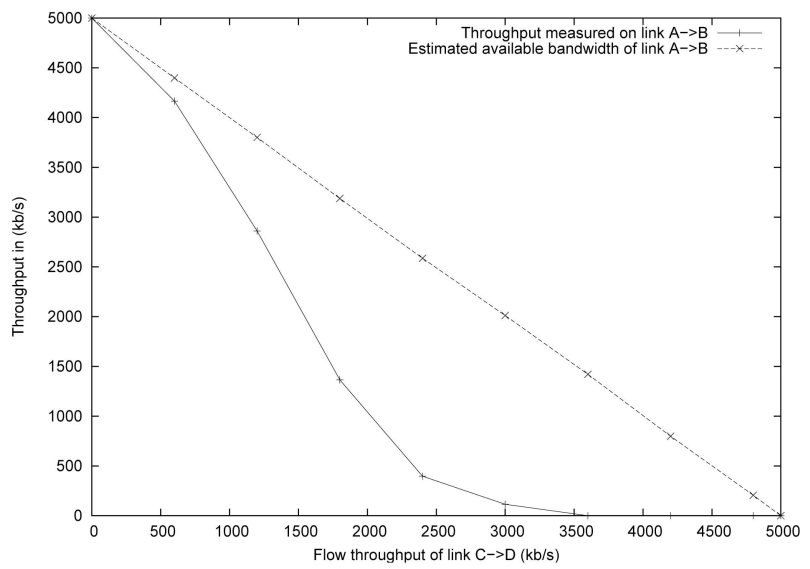

(b)

Fig. 2. Available bandwidth in the scenario in Fig. 1 (NS-2 simulation results). (a) Capacity of 2 Mbps. (b) Capacity of 11 Mbps.

capacity of the radio medium minus the bandwidth consumed by the flow on link (C, D).

The second curve of these figures, denoted by "Throughput measured on link (A, B)," represents the true available bandwidth on link (A, B), which corresponds to the maximum throughput that can be actually transmitted on the link. We notice that increasing the throughput of link $(C, D)$ accentuates the difference between the estimation and the real available bandwidth on link (A, B).

This difference can be explained by the presence of collisions occurring at node $\mathrm{B}$. These collisions lead to an important throughput decrease on link (A, B), which none of the aforementioned estimators manages to predict. Therefore, it is essential not only to consider the total amount of traffic emitted in each contention zone but also to take at least into account collisions.

\section{Improving AvaILABLE BANDWIDTH Estimation ACCURACY}

Based on the previous literature study and considering how the IEEE 802.11 MAC protocol operates, we can point out a few phenomena that may have an influence on the bandwidth available from a node to one of its neighbors:

- The carrier sense mechanism prevents two close emitters from transmitting simultaneously, unless they draw the same backoff counter value. Therefore, an emitter shares the channel bandwidth with all its close neighbors. The channel utilization has to be monitored to evaluate the capacity of a node to emit a given traffic volume.

- For a transmission to take place, the receiver needs that no interference occurs during the whole transmission. Therefore, the value of the available bandwidth on a link depends on both peer channel utilization ratios and also on the idle period synchronization. This synchronization needs to be evaluated.

- No collision detection is possible in a wireless environment. Therefore, whenever a collision happens, both colliding frames are completely emitted, maximizing the bandwidth loss. As shown by the scenario depicted in Fig. 1, the collision probability needs to be estimated and integrated to the ABE.

- Finally, when collisions happen on unicast frames, the IEEE 802.11 protocol automatically retries to emit the same frame, drawing the backoff counter in a double-sized contention window. The time lost in the additional overhead may also have an impact on the available bandwidth and has to be evaluated.

In this section, we examine in turn all four points listed above and describe how we take these phenomena into account. Each of this point can be evaluated with more or less accuracy, depending on which set of nodes is implied in the evaluation. However, if involving several nodes in the evaluation of a single parameter may improve exactness, it often requires additional communications. Each of the following paragraphs describes what we think represents the best compromise between precision and induced load.

\subsection{Carrier Sense Mechanism: Estimating a Node's Emission Capabilities}

Whenever a node needs to send a frame, it first needs to contend for medium access and it cannot emit its frame unless the medium is free. Therefore, a potential sender needs to evaluate the load of the medium, i.e., the proportion of time the medium is idle to determine the chance it has to successfully gain access to the shared resource. Such evaluation is also performed by the solutions proposed in [7] or [11].

Let us consider a node $s$ in the network during an observation interval of $\Delta$ seconds. We use the following notations:

- $\quad T_{\text {idle }}(s)$ is the total idle time, i.e., the total time during which node $s$ neither emits any frame nor senses the medium busy. Both physical and virtual carrier sense mechanisms should report an idle state. This includes periods during which no frame is ready to be emitted as well as periods of deferral (backoff time and interframe spacing). 


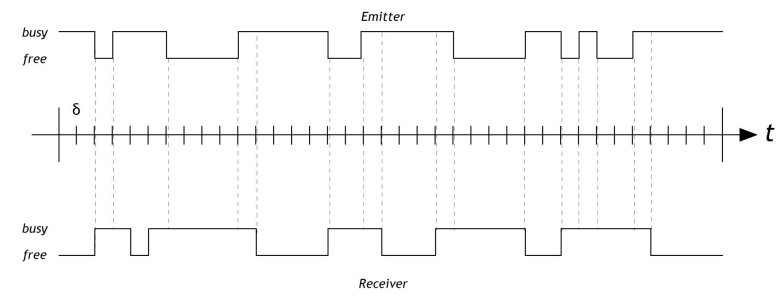

Fig. 3. Medium idle periods of sender and receiver that never overlap.

- $B_{s}$ is the bandwidth available to node $s$, i.e., the maximum throughput it can emit without degrading close flow's rate.

- $C_{\max }$ is the capacity of the medium.

During an arbitrary observation interval $\Delta$, each node may monitor the radio medium in its surroundings and measure the total amount of time $T_{\text {idle }}$ that is idle for emitting frames. To adapt the evaluation to the MAC protocol's behavior, periods of time shorter than IEEE 802.11's DIFS timing shall not be added to the total idle time count, as such intervals do not allow any backoff decrease nor medium access. As the medium is considered busy as soon as a signal above the carrier sensing threshold is received, this method does not only take into account the bandwidth used in the transmission range of the nodes but also in the whole carrier sensing area.

As this monitoring neither takes into account the IEEE 802.11 's variable overhead nor the reception side of the transmission, the available bandwidth computed by this method at node $s$ is imprecise. However, it provides a threshold above which the medium access probability decreases rapidly. Some frames may still be successfully emitted, though, due to a favorable scheduling of transmissions or to capture effects. As long as the medium load remains below this threshold, a scheduling between different contending emitters preventing two simultaneous emissions exists. We therefore consider that this value is an upper bound of the available bandwidth we are seeking:

$$
B_{s} \leq \frac{T_{\text {idle }}(s)}{\Delta} \cdot C_{\text {max }} .
$$

The reader should note that the value of $C_{\max }$ shall not represent the raw medium capacity, as advertised by the standard, but must take into account the fixed overhead (headers, acknowledgments,...) introduced by the MAC protocol. For example, a 54-Mbps implementation of IEEE 802.11 cannot deliver throughputs higher than 33.2 Mbps.

\subsection{Idle Period Synchronization: Estimating a Link's Available Bandwidth}

This part has been first presented in [15], and its key ideas are included in this paper for a better legibility. In Section 3.1, we have evaluated an upper bound of the available bandwidth a node could use to emit frames. The reception part of the transmission also requires the medium to be free during the transmission, and thus, the previous measurement should also be considered at the receiver's side.

Let us simply consider a radio link composed of two neighbor nodes $s$ and $r$. In order to use combinatorial tools,

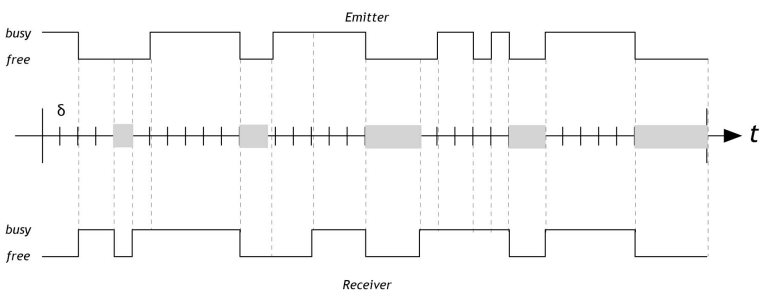

Fig. 4. Medium idle periods of sender and receiver that overlap.

we consider that time is discrete. We introduce the following additional notations:

- $\delta$ is the time sampling step, referred to, hereafter, as the time unit.

- $\tau_{m}=\Delta / \delta$ is the number of time units in a measurement period.

- $\tau_{s}$ (respectively $\tau_{r}$ ) is the number of time units during which the medium is available for node $s$ (respectively $r$ ) in a measurement period, computed according to the constraints described above.

- $B_{s}$ (respectively $B_{r}$ ) is the available bandwidth bound for node $s$ (respectively $r$ ), measured with the method described in Section 3.1.

- $B_{(s, r)}$ is the true available bandwidth on link $(s, r)$, i.e., the real bandwidth that can be achieved without degrading close flows.

- $b_{(s, r)}$ is the estimated available bandwidth on link $(s, r)$.

If $B_{s}$ is null or close to zero, $s$ either never gains access to the medium or already emits frames at a rate that saturates the radio medium. Similarly, if the medium is always busy on the receiver's side, the frames systematically experience collisions and the communication never succeeds. Trivially, we can state that $B_{(s, r)} \leq \min \left(B_{s}, B_{r}\right)$. However, if sending a flow with a throughput higher than $\min \left(B_{s}, B_{r}\right)$ necessarily provokes a medium saturation around $s$ and/or $r$, considering this minimum value as the available bandwidth may also lead to an overestimation, as, in the general case, idle periods at emitter's and receiver's sides are desynchronized.

As an example, Figs. 3 and 4 represent the medium availability during the time at the emitter and the receiver sides of a given transmission. In both situations, the idle time values measured at each node by the previously described mechanism are similar and the difference between both scenarios is a small shift of time. In Fig. 3, the periods of medium availability of both peers never overlap and the available bandwidth on the link is null. In the opposite case, the scenario depicted in Fig. 4 offers several communication opportunities on the link, represented by grayed areas.

In ad hoc networks, due to the complex interactions between nodes, emitters and receivers are unlikely to be perfectly synchronized. Precisely evaluating the impact of this asynchronism requires the exchange of the exact medium utilization patterns of both peers and a fine clock synchronization mechanism, which represents a huge overhead, though. Therefore, we propose to use a probabilistic mechanism to estimate the effect of this phenomenon.

Let us examine the requirements for a successful frame transmission. First, for the communication to start, the 


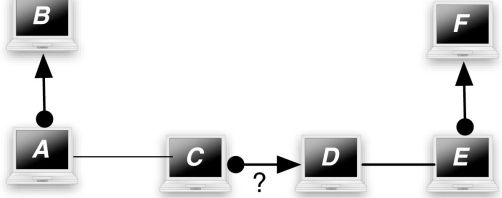

Fig. 5. Scenario illustrating link synchronization phenomenon.

medium has to be free during at least DIFS on the emitter's side so that this emitter gains access to the medium. Once the emission has started, the status of the medium at the emitter's side is irrelevant. On the receiver's side, the medium has to be free during the time required to transmit the whole data frame $\left(T_{\mathrm{DATA}}\right)$; otherwise, a collision occurs. This value is not perfectly accurate, though. It makes the hypothesis that the level of signal that would provoke a collision is equal to the carrier sense threshold, regardless of the distance between the emitter and the receiver, for example. It also does not take into account the propagation time.

Let us consider a uniform random distribution of the medium occupancy over the observation period. It is then possible to compute the expected delay $E\left(l_{(r, s)}\right)$ before nodes $s$ and $r$ sense the medium idle simultaneously. We denote by $p(i, j, k)$ the probability that

- the first occurrence of such a synchronization in a measurement interval occurs at time slot $i$,

- the sender has been idle for $j$ time units before synchronization,

- the receiver has been idle for $k$ time units before synchronization.

Then,

$$
p(i, j, k)=\frac{\left(\begin{array}{c}
i \\
j
\end{array}\right) \cdot\left(\begin{array}{c}
i-j \\
k
\end{array}\right) \cdot\left(\begin{array}{c}
\tau_{m}-i-1 \\
\tau_{s}-j-1
\end{array}\right) \cdot\left(\begin{array}{c}
\tau_{m}-i-1 \\
\tau_{r}-k-1
\end{array}\right)}{\left(\begin{array}{c}
\tau_{m} \\
\tau_{s}
\end{array}\right) \cdot\left(\begin{array}{c}
\tau_{m} \\
\tau_{r}
\end{array}\right)} .
$$

From this expression, we can compute the probability $P\left(l_{(s, r)}=i\right)$ that the first synchronization occurs at a given time unit and the expected delay $E\left(l_{(s, r)}\right)$ before synchronization:

$$
\begin{aligned}
P\left(l_{(s, r)}=i\right) & =\sum_{j=\max \left(0, \tau_{s}-\left(\tau_{m}-i\right)\right)}^{\min \left(\tau_{s}-1, i-1\right)}\left(\sum_{k=\max \left(0, \tau_{r}-\left(\tau_{m}-i\right)\right)}^{\min \left(\tau_{r}-1, i-1-j\right)} p(i, j, k)\right), \\
E\left(l_{(s, r)}\right) & =\sum_{i=0}^{\min \left(\tau_{m}, 2 \cdot \tau_{m}-\left(\tau_{s}+\tau_{r}\right)\right)} i \cdot P\left(l_{(s, r)}=i\right) .
\end{aligned}
$$

Still considering a uniform random distribution of the medium occupancy, the available expected bandwidth $E\left(b_{(s, r)}\right)$ can be evaluated by expressing the probability that the medium is free simultaneously at the emitter's and receiver's sides:

$$
\begin{aligned}
\mathrm{P}\left(b_{(s, r)}=i\right) & =\frac{\left(\begin{array}{c}
\tau_{s} \\
i
\end{array}\right) \cdot\left(\begin{array}{c}
\tau_{m}-\tau_{s} \\
\tau_{r}-i
\end{array}\right)}{\left(\begin{array}{c}
\tau_{m} \\
\tau_{r}
\end{array}\right)}, \\
\mathrm{E}\left(b_{(s, r)}\right) & =\sum_{i=0}^{\min \left(\tau_{s}, \tau_{r}\right)} i \cdot P\left(b_{(s, r)}=i\right)=\tau_{s} \times \tau_{r} .
\end{aligned}
$$

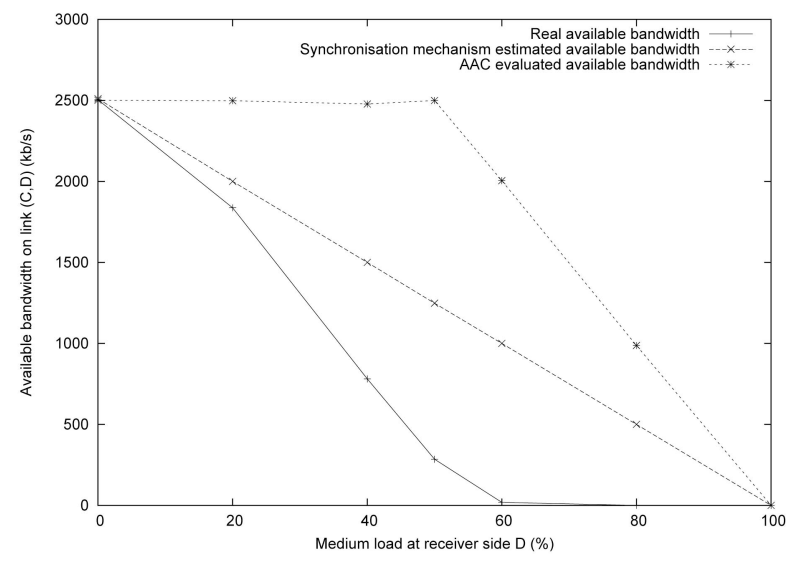

Fig. 6. Available bandwidth for the link synchronization scenario.

To illustrate the importance of this synchronization phenomenon, let us consider the scenario shown in Fig. 5. Communications are represented by arrows and nodes in mutual carrier-sense range are linked with a dashed line. If no line joins two nodes, they are totally independent.

We performed simulations using the NS-2 simulator. When no medium access layer modification is performed, the simulated medium capacity can be set to 2 or $11 \mathrm{Mbps}$, resulting, respectively, in a 1.6- and 5-Mbps maximum application-layer throughput.

Nodes C and D evaluate the available bandwidth on link $(C, D)$ and this value evolves with the throughput of the $(\mathrm{E}, \mathrm{F})$ flow. The $(\mathrm{A}, \mathrm{B})$ flow constantly uses 50 percent of the medium capacity (i.e., 800 kilobits per second (Kbps) at $2 \mathrm{Mbps}$ and $2500 \mathrm{Kbps}$ at $11 \mathrm{Mbps}$ ).

Fig. 6 represents the real available bandwidth on link (C, D), measured by adding a flow between both nodes and by evaluating its maximum achieved throughput that does not degrade the two existing flows (A, B) and (E, F). As an example, we compare this value with the available bandwidths estimated by the AAC protocol described above and by the mechanism taking synchronization into account.

AAC considers that the link available bandwidth is equal to the minimum of the node available bandwidths of the two link extremities and does not consider synchronization between the sender and the receiver. Hence, it overestimates the real available bandwidth on the link. As shown in the figure, we can see that considering the synchronization drastically enhances the estimation quality. However, it still leads to an overestimation of the available bandwidth. Indeed, considering a uniform distribution of the silence periods is an approximation that does not always reflect the scenario details. Considering another type of idle period distribution would not lead to better results in the general case, though. Finally, with this estimation, collisions are not taken into account.

Therefore, if this mechanism achieves a better approximation, it still leads to a certain level of inaccuracy. In the subsequent sections, we will try to refine this estimation using other easily obtainable data. 


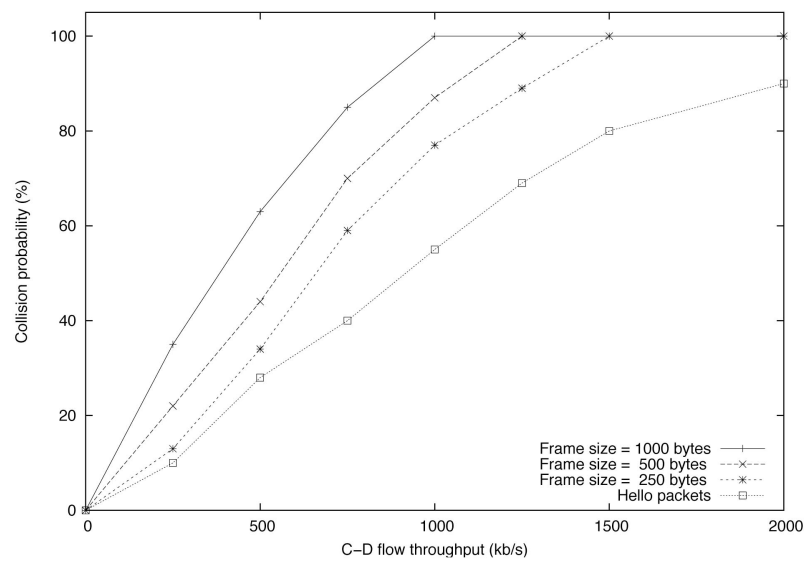

(a)

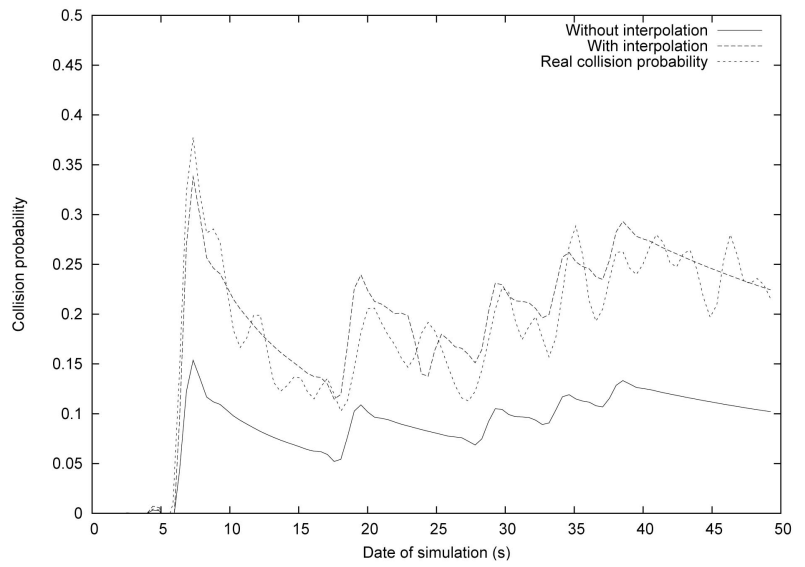

(b)

Fig. 7. Interpolated collision probability. (a) Collision probability on node B (cf. Fig. 1) obtained by simulation. (b) Precision of the interpolated collision probability on a random topology.

\subsection{Taking Collisions into Account}

The use of the previous probabilistic estimation still leads to a certain level of inaccuracy. Indeed, there is a chance, even for a single frame that, when a packet is emitted, the medium is not idle at the receiver's side, provoking a collision. A typical example of such a situation is the configuration depicted in Fig. 1. In this situation, protocols like BRuIT, CACP, or AAC overestimate the available bandwidth. The difference between the evaluated and real available bandwidths is due to repeated collisions at node B. This phenomenon has to be evaluated, but how can we estimate collision probability without introducing explicit probe packets?

Emitters can evaluate the collision probabilities toward certain receivers by counting the number of retransmission events at the MAC layer. However, such strategy is only applicable to nodes already emitting data frames. The evaluation mechanism should, however, be active even when no data traffic is emitted.

Several routing protocols use Hello packets, regularly emitted by every nodes, to exchange connectivity and bandwidth-related information. A collision probability may be computed on the basis of these Hello packets. When such packets are emitted regularly, a receiver may estimate the amount of Hello packets it should receive in a given time interval. Comparing this number with the effective number of received Hello packets gives an estimation of the collision probability between both peers. Such technique may confuse congestion-related effects with losses due to collisions, though. However, when a node does not succeed in emitting as many Hello packets as it should due to an overloaded medium, its own available bandwidth upper bound is already low. However, when a node does not succeed in emitting most of its Hello packets due to a very overloaded medium, its own available bandwidth upper bound is already low. Therefore, trying to increase further the accuracy of the evaluation by considering the collision probability does not represent a valuable enhancement. Another strategy could base the computation of the collision probability on the Hello packet sequence numbers.
However, such strategy increases the required memory and may be unsuited in dense networks.

Another source of imprecision comes from the size of these control packets. In a slowly evolving neighborhood, successive Hello packets are of comparable sizes. As a consequence, the computed collision probability may not be representative for small or big data frames. To address this issue, we extend the measurement by computing the Lagrange interpolating polynomial fitting the data. Let us designate by $p_{\text {hello }}$ the collision probability computed on Hello packets. If we denote by $f(m)$ this polynomial, the collision probability $p_{m}$ for packets of $m$ bits is approximated by $p_{m}=f(m) \cdot p_{\text {hello }}$.

Let us consider the scenario depicted in Fig. 1. Fig. 7a shows the results of the simulations performed with NS-2 to obtain the collision probability on node B for different packet sizes and for Hello packets. From these measurements, we can deduce the interpolated polynomial corresponding to this situation: $f(m)=-5.65 \cdot 10^{-9} \cdot m^{3}+$ $11.27 \cdot 10^{-6} \cdot m^{2}-5.58 \cdot 10^{-3} \cdot m+2.19$.

This Lagrange polynomial being computed on a particular scenario does not reflect the evolution of the probability in the general case. Distributed computation of the coefficients of such polynomial may be possible and is the subject of future work. However, we evaluated its accuracy in random topologies. Fig. $7 \mathrm{~b}$ shows such results in a scenario involving 10 nodes and five CBR connections between random sources and destinations, with random throughputs and composed of 1,000-byte frames. It depicts the collision probability evolution with time in three situations. The lowest graph represents the probability of collision of Hello packets. The two upper curves represent this probability rescaled with the aforementioned polynomial and the real collision probability. Both results present some differences but are close, which indicate that this interpolation is both efficient and necessary.

It is important to note that the collision probability depends on the packet size and on the distribution of the medium occupancy at the receiver's side. Up to here, the bandwidth evaluation method we propose combines passive measurements with piggybacking of the information in 
Hello messages. The originality of this proposition is on the deduction of an accurate expected collision probability from online measurements and offline computations.

It can be further enhanced, though. When a node experiences a collision, it doubles its contention window size. Until now, we have considered the proportion of bandwidth lost due to the collisions themselves but not to the additional overhead introduced by the binary exponential backoff mechanism.

\subsection{Taking the Backoff into Account}

The time spent in the IEEE 802.11 binary exponential backoff procedure depends on the version of the protocol and on the amount of collisions on the link. It is independent of the frame size and may become large compared to the time required to transmit frames. Therefore, when transmitting small frames, ignoring the influence of this backoff introduces a high inaccuracy in the estimated available bandwidth.

First, let us consider that there is no collision. The backoff is drawn according to a uniform law in the interval $\left[0 ; C W_{\min }-1\right]$, where $C W_{\min }$ is defined by the MAC protocol specification. On a large observation window, the backoff can be approximated by its average value $\frac{C W_{\min }-1}{2}$. When collisions happen, the exponential backoff mechanism is triggered. After each unsuccessful transmission, the contention window size is doubled up to a maximum value denoted by $C W_{\max }$. In this situation, the average backoff value increases above $\frac{C W_{\min }-1}{2}$ and it is necessary to model the time consumed by the exponential backoff process.

Let us consider that an arbitrary wireless link suffers from collisions with a probability $p$. In this evaluation, we make the same assumption as [16]: $p$ is supposed to be independent of the sender's contention window size. The collision probability thus reflects the probability that a frame, once emitted, suffers a collision. This is an approximation, as a sender suffering a collision probably provoked a collision itself, triggering the collision avoidance mechanism at another emitter. This other emitter increases simultaneously its contention window, resulting in a reduced collision probability for successive retransmission attempts. We neglect this effect, though, as its influence decreases with the number of contending emitters and as the collision probability is regularly updated by the mechanism described in the previous section.

For every frame, the transmission is successful at the first attempt with probability $(1-p)$. It succeeds at the second attempt with probability $p \cdot(1-p)$. After $C$ unsuccessful retransmission attempts, the IEEE 802.11 standard specifies that the frame should be dropped, resulting in at most $C+1$ transmissions of the same frame.

Let us denote by $X$ the random variable representing the number of retransmissions suffered by a given frame. We can notice that the success or the failure of the $C$ th retransmission attempt has no influence on the available bandwidth, the medium being occupied for the whole duration of the frame in both cases. Therefore, this variable's probability law can be expressed by

$$
\left\{\begin{array}{l}
\forall k \in[0 ; C-1], P(X=k)=p^{k} \cdot(1-p), \\
P(X=C)=p^{C} \\
P(X>C)=0
\end{array}\right.
$$

Using this expression, we can evaluate the number of backoff slots decremented on average for a single frame:

$$
\overline{\text { backoff }}=\sum_{k=0}^{C} P(X=k) \cdot \frac{\min \left(C W_{\max } ; 2^{k} \cdot C W_{\min }\right)-1}{2} .
$$

To simplify the expression, let us define $M$ so that $C W_{\max }=2^{M} \cdot C W_{\min }$ with $M \leq C$. If we further replace $P(X=k)$ by its expression, we get

$$
\begin{aligned}
& \overline{\text { backoff }}=\left(\sum_{k=0}^{M} p^{k} \cdot(1-p) \cdot \frac{2^{k} \cdot C W_{\min }-1}{2}\right) \\
& +\left(\left(\sum_{k=M+1}^{C-1} p^{k} \cdot(1-p)\right)+p^{C}\right) \cdot \frac{C W_{\max }-1}{2}, \\
& \overline{\text { backoff }}=\frac{(1-p) \cdot C W_{\min }}{2} \cdot\left(\sum_{k=0}^{M}(2 \cdot p)^{k}\right)-\frac{(1-p)}{2} \cdot\left(\sum_{k=0}^{M} p^{k}\right) \\
& +\frac{\left(C W_{\max }-1\right) \cdot(1-p)}{2} \cdot\left(\sum_{k=M+1}^{C-1} p^{k}+\frac{p^{C}}{1-p}\right) \text {. }
\end{aligned}
$$

Replacing $\sum_{k=a}^{b} p^{k}$ by $\frac{p^{a}-p^{b-a+1}}{1-p}$, we obtain the following formula:

$$
\begin{aligned}
\overline{\text { backoff }=} & \frac{(1-p) \cdot C W_{\min }}{2} \cdot \frac{1-(2 \cdot p)^{M+1}}{1-2 \cdot p}-\frac{(1-p)}{2} \\
& \cdot \frac{1-p^{M+1}}{1-p}+\frac{\left(C W_{\max }-1\right) \cdot(1-p)}{2} \\
& \cdot\left(\frac{p^{M+1}-p^{C-M-1}}{1-p}+\frac{p^{C}}{1-p}\right), \\
\overline{\text { backoff }=} & \frac{C W_{\min } \cdot(1-p) \cdot\left(1-(2 \cdot p)^{M+1}\right)}{2 \cdot(1-2 \cdot p)}+\frac{1}{2} \\
& \cdot\left(p^{M+1}-1+\left(C W_{\max }-1\right) \cdot\left(p^{M+1}-p^{C-M-1}+p^{C}\right)\right) .
\end{aligned}
$$

Let us denote by $K$ the proportion of bandwidth consumed by the backoff mechanism when collisions happen and by $T(m)$ the time separating the emission of two consecutive frames. This delay essentially depends on the emission rate and on the frame size $m$. Then, $K$ can be expressed by

$$
K=\frac{D I F S+\overline{\text { backoff }}}{T(m)} .
$$

\subsection{ABE: Available Bandwidth Estimation}

The different points mentioned above can be combined to estimate the available bandwidth on a wireless "link," i.e., between a given emitter and a given receiver. The whole mechanism, called $\mathrm{ABE}$, leads to a lightweight protocol design, as it mainly relies on the perception that nodes have of their immediate environment. To summarize, the available bandwidth between two neighbor nodes $s$ and $r$ can be estimated by the following equation:

$$
E_{\text {final }}\left(b_{(s, r)}\right)=(1-K) \cdot(1-p) \cdot E\left(b_{(s, r)}\right),
$$

where $E\left(b_{(s, r)}\right)$ is the available bandwidth on link $(s, r)$ evaluated by monitoring the radio channel and combining emitter and receiver's values in a probabilistic manner, $p$ is the collision probability measured on the 


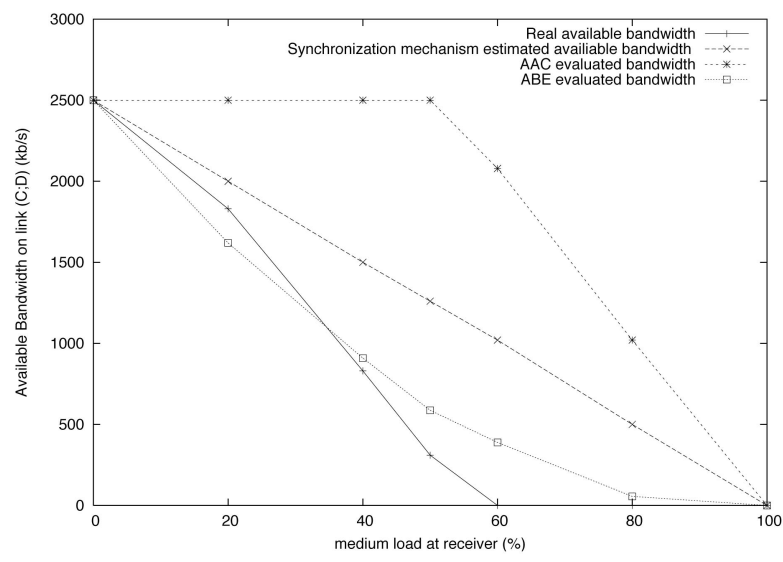

Fig. 8. Available bandwidth for the link synchronization scenario, considering collisions' impact.

received Hello packets and rescaled to the appropriate packet size, and $K$ is the proportion of bandwidth lost due to the backoff scheme computed due to $p$.

Fig. 8 represents the results obtained on the scenario in Fig. 5 when taking into account the previously described techniques.

\section{ImPLemEntation Setup}

It is quite tricky, from an operational point of view, to evaluate the performance of the sole ABE part of an existing QoS protocol. Therefore, for comparison purposes, we have integrated the previously described bandwidth evaluation technique $\mathrm{ABE}$ into a protocol and implemented it under NS-2. This simulator has been chosen because of the availability of other protocol models. We have chosen to integrate $\mathrm{ABE}$ into $\mathrm{AODV}$, in order to be similar to BRuIT, QoS-AODV, or AAC. It is thus based on broadcasted route request messages, admission control at each intermediate node, and explicit reservation by a unicast route reply message issued by the destination. We can thus study the impact of our estimation technique on the bandwidth management in the network by comparing the performance of the different protocols. The protocol is called hereafter ABE-AODV.

\subsection{ABE Features}

In ABE-AODV, neighboring nodes exchange their available bandwidth computed locally via Hello messages. Every $\Delta$ seconds, each node locally estimates its medium occupancy ratio and includes this information in a Hello packet. These values are then converted into link evaluations using (3), as mentioned in Section 3.5.

The accuracy of the bandwidth evaluation obviously depends on the value of $\Delta$, which can be considered as a sampling period. The larger $\Delta$ is, the more stable the measurements will be, hiding fast variations in the medium load. However, $\Delta$ should also be small enough to allow fast reactions to long-term load variations and to node mobility.

Hello-based techniques generate additional overhead depending on the Hello emission frequency. Ideally, the Hello packet emission frequency should be adapted to the node mobility and/or to the flow dynamics. In order to have meaningful comparisons, we chose to fix this value to $\Delta=1$ second in ABE-AODV. Similarly, all compared protocols are tuned accordingly to emit one information frame each second.

\subsection{Integration into AODV: Admission Control}

We have slightly modified AODV in order to transform it into a QoS protocol based on ABE. It thus becomes a cross-layer routing protocol. The MAC layer estimates proactively and periodically the available bandwidth of the neighboring links, and the routing layer is in charge of discovering QoS routes complying to the application demands, basing its decisions on the MAC layer information.

\subsubsection{Route Discovery}

The aim of the route discovery procedure is to find a route between the sender and the receiver that meets the constraints specified by the application level in terms of bandwidth. Therefore, two flows with the same source and destination can follow different routes depending on the network state.

When a source node has data to send, it broadcasts a route request $(R R E Q)$ to its neighbors. The $R R E Q$ packet contains the address of the sender, the channel use, the requirements at the application level, the destination address, and a sequence number. Each mobile node that receives such an $R R E Q$ performs an admission control by simply comparing the bandwidth requirement carried in the $R R E Q$ packet to the estimated available bandwidth on the link it received the $R R E Q$ on. If this check is positive, the node adds its own address to the route and forwards the $R R E Q$; otherwise, it silently discards the message. This step is different from the other tested protocols as the admission control is done at the receiver side and not at the sender side. This is explained by the fact that, in ABE, each node stores the available bandwidths of its ingoing links. Finally, if the destination receives a first $R R E Q$, it sends a unicast route reply $(R R E P)$ to the initiator of the request along the reverse path. The resources are then reserved and the new QoS flow can be sent.

\subsubsection{Intraflow Contention Problem}

Simply comparing the bandwidth application requirement and a link available bandwidth is not sufficient to decide about the network ability to convey a flow. Indeed, the intraflow contention problem has to be considered when performing multihop admission control.

In [12], the authors compute a value called contention count (CC) of a node along a given path. This value is equal to the number of nodes on the multihop path that are located within the carrier sensing range of the considered node. To calculate the CC of each node, the authors analyze the distribution of the signal power.

As in [17], for simplicity reasons, in $\mathrm{ABE}$, we rather use a direct relationship between the end-to-end throughput and the number of hops. Hence, after consideration of the intraflow contention on an intermediate node $j$, which is located at $H$ hops from the source and has received the 
TABLE 1

General Parameters for Simulations Presented in This Paper

\begin{tabular}{|c|c|}
\hline Parameters & Values \\
\hline \hline HELLO interval & $1 \mathrm{~s}$ \\
\hline Packets size & 1000 bytes \\
\hline Medium capacity & $2 \mathrm{Mb} / \mathrm{s}$ or $11 \mathrm{Mb} / \mathrm{s}$ \\
\hline Communication range $(2 \mathrm{Mb} / \mathrm{s})$ & $250 \mathrm{~m}$ \\
\hline Carrier sensing range $(2 \mathrm{Mb} / \mathrm{s})$ & $550 \mathrm{~m}$ \\
\hline Communication range $(11 \mathrm{Mb} / \mathrm{s})$ & $160 \mathrm{~m}$ \\
\hline Carrier sensing range $(11 \mathrm{Mb} / \mathrm{s})$ & $350 \mathrm{~m}$ \\
\hline Grid Size & $1000 \mathrm{~m} \times 1000 \mathrm{~m}$ \\
\hline C (Number of retransmissions) & 6 \\
\hline
\end{tabular}

$R R E Q$ from a node $i$, the available bandwidth considered for admission control, denoted by $B(i, j)$, is equal to

$$
B(i, j)=\frac{E_{\text {final }}\left(b_{(i, j)}\right)}{\min (H, 4)},
$$

where $E_{\text {final }}\left(b_{(i, j)}\right)$ is the available bandwidth of link $(i, j)$ as computed by ABE (3).

\section{Simulations}

This section presents a few simulation results that we found representative of the general behavior of the different protocols. We compare the performances of our estimation technique through ABE-AODV with three other protocols available on the Web and described in Section 2: QoS-AODV, AAC, and BRuIT. ${ }^{1}$ Simulations were performed using NS-2 version 2.27 and use the MAC layer implementation provided with the simulator. Other simulation parameters are given in Table 1. Each graph included below represents the average results obtained over 30 simulations using different random seeds.

We performed several simulations with different parameters such as network load, flow maximum throughput, number of nodes, and network diameter. A few general results apply to all simulated protocols. For instance, when the network load is low, all protocols yield similar results, even the non-QoS-enabled ones. Flow QoS degradation and false admission control phenomena begin to appear when congestion rises above a certain level. On the opposite, when the network load is too high or when the flows' individual throughputs are too important, very few flows are admitted. In this case, the problem falls back to the sole intraflow contention problem for which all solutions have similar answers.

\subsection{Admission Control Mechanism Accuracy}

\subsubsection{Single-Hop Communications}

First of all, to illustrate the accuracy of ABE, we chose to focus on a small static network involving 10 randomly positioned nodes. The channel capacity is set to $11 \mathrm{Mbps}$ in this example and five one-hop CBR connections are established in the network. For each flow, the source is

1. BRuIT: http://perso.ens-lyon.fr/isabelle.guerin-lassous/QoS.html. QoS-AODV and AAC: http://www.ctr.kcl.ac.uk/members/ronan/ default.asp. randomly chosen and the destination is also randomly chosen among the source's neighbors. Nodes are not in the same contention area, but we only consider one-hop flows, leaving intraflow contention problems aside for now.

Each simulation lasts 50 seconds and one flow is started every 5 seconds. Fig. 9 represents the evolution of the different flow throughputs as a function of the simulation time for all solutions. We can notice that when no admission control is present, some flows suffer from medium overload. For instance, flow 1, whose throughput is stable at the beginning, experiences an irregular performance after the beginning of flow 4 . This confirms that the channel is not able to sustain this scenario's total traffic and that problems start to appear after the admission of flow 4 .

AAC, QoS-AODV, and BRuIT tend to overestimate the available bandwidth. Therefore, admission control mechanism is not severe enough and flow 4 is accepted, resulting in a similar instability. In this scenario, overestimation is due to the fact that these protocols do not take into account collisions.

ABE-AODV's admission control accepts all flows except the fourth one. As throughputs are stable, all four remaining flows are able to fit into the network, indicating that, for other scenarios, the fourth flow was the only cause of overload.

\subsubsection{Multihop Flows}

Let us now illustrate the case of multihop flows. In the chosen scenario, 20 nodes are randomly positioned in the simulation square. Seven CBR connections are established. Each of these flows is composed of 1,000-byte frames, and all flow throughputs are randomly drawn between 0 and $300 \mathrm{Kbps}$ for a 2-Mbps medium capacity. ${ }^{2}$ Flow sources and destinations are chosen randomly and independently requiring that flows are routed across multihop paths. The simulation lasts 50 seconds and one flow is started every 5 seconds.

Fig. 10a shows the throughput of the seven flows when no admission control is performed. Once again the network becomes really congested after the appearance of the fourth flow (after 20 seconds), even though some irregularities appear when the third flow begins.

Figs. $10 \mathrm{~b}$ and $10 \mathrm{c}$ represent the throughput of all flows when AAC and QoS-AODV are used. Similar behavior can be noticed, admission control being too permissive in this situation, indicating that the available bandwidth is overestimated.

Fig. 10d presents the results obtained with BRuIT. On the opposite, only the first flow is admitted. ABE-AODV admits three flows that all meet their expected performance. In this situation, BRuIT is, on the opposite, too severe with flows, which was not its behavior in the previous setup. BRuIT has a different way of computing intraflow contention, multiplying the required bandwidth by 5 instead of 4 , considering that all two-hop neighbors are prevented from emitting when a node transmits a flow. These results, compared to the results obtained by the other protocols, indicate that a certain level of spatial reuse is missed by this approach.

2. The channel capacity differs from the first scenario to illustrate that the estimator is efficient at different throughputs. 


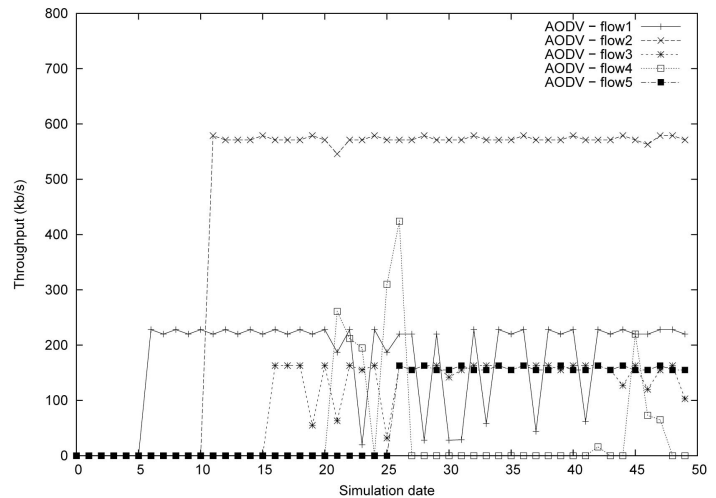

(a)

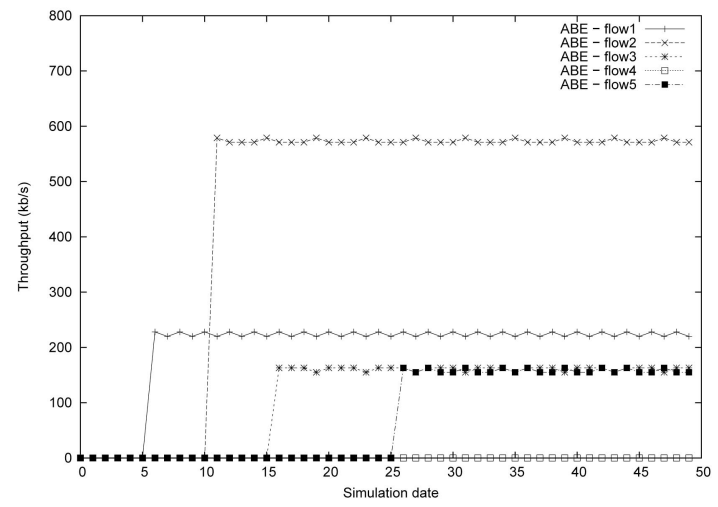

(c)

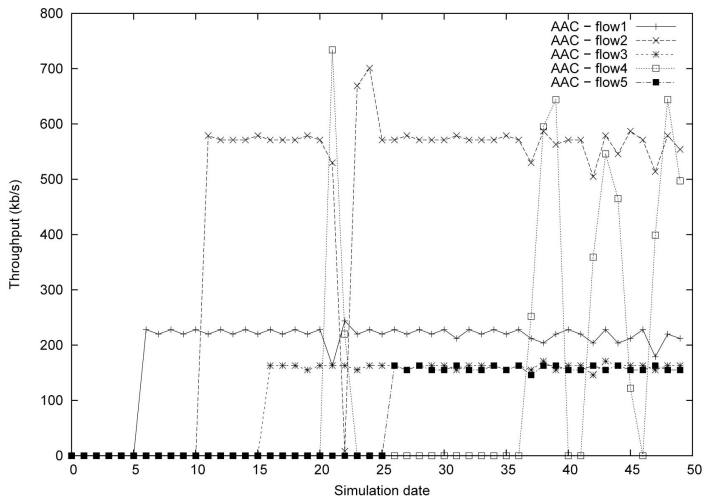

(b)

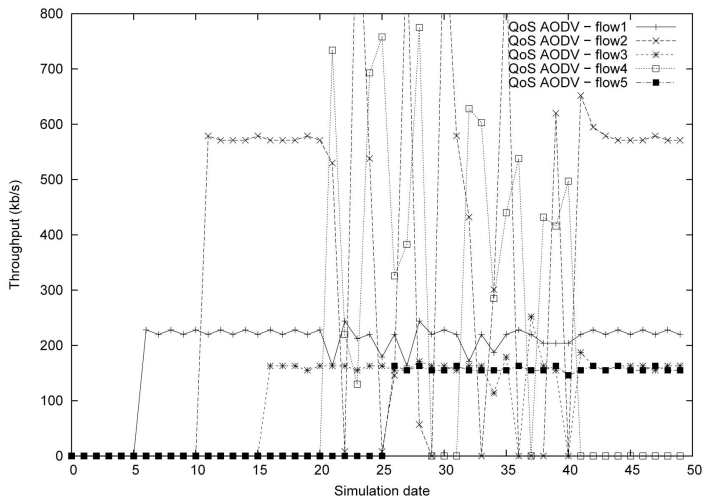

(d)

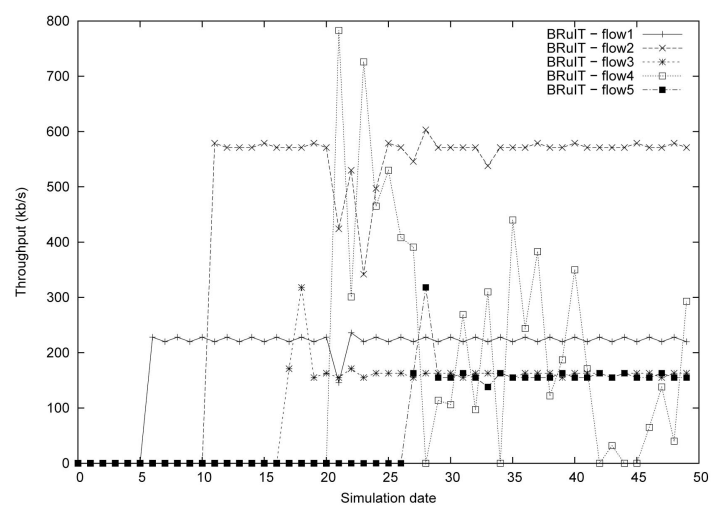

(e)

Fig. 9. Throughput of each one-hop flow using AODV, AAC, QoS-AODV, BRuIT, and ABE-AODV (11-Mbps medium capacity). (a) AODV. (b) AAC. (c) ABE-AODV. (d) QoS-AODV. (e) BRulT.

\subsection{Statistical Results}

To indicate the accuracy of the bandwidth estimator in more general cases, we performed a set of simulations with random number of nodes, location of these nodes, flow sources, flow destinations, and flow throughputs. In these scenarios, we measured the proportion of flows that were admitted in the network and that achieve a throughput higher than 95 percent of their request. This metric is able to reflect, in the general case, the accuracy of the bandwidth evaluation mechanism. When a flow is admitted and does not fit into the network, it either achieves a lower throughput than its request or provokes a degradation of close flows. In both cases, the number of correctly admitted flow decreases.
Fig. 11 represents the proportion of correctly admitted flows, $\beta$, i.e., the ratio between the number of flows suffering no degradation over the total amount of requests. To produce this particular graph, simulations were performed on network sizes ranging from 10 to 40 nodes. The medium capacity was set to $11 \mathrm{Mbps}$ and five CBR connections were established, asking for throughputs uniformly drawn between 0 and $500 \mathrm{Kbps}$.

All protocols see a decrease in the $\beta$ value as the number of nodes gets higher. This is mainly due to the availability of alternate routes for which information is not fully accurate. When the network is too sparse, situations in which nodes interfere without being able to communicate are more frequent. As the network gets denser, collisions between 


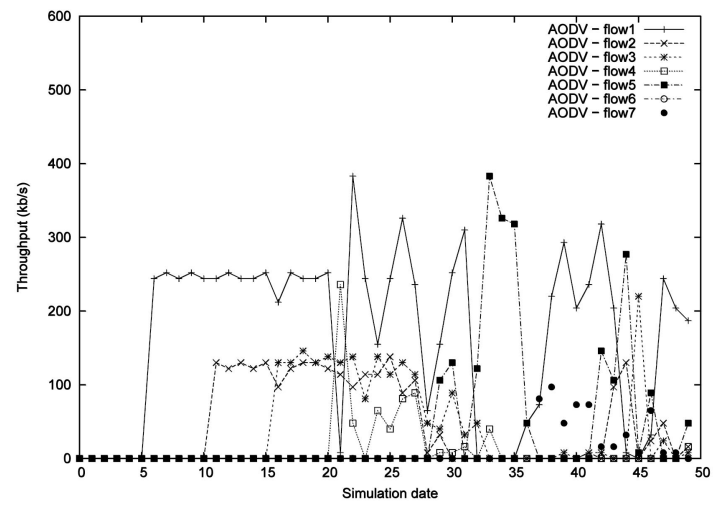

(a)

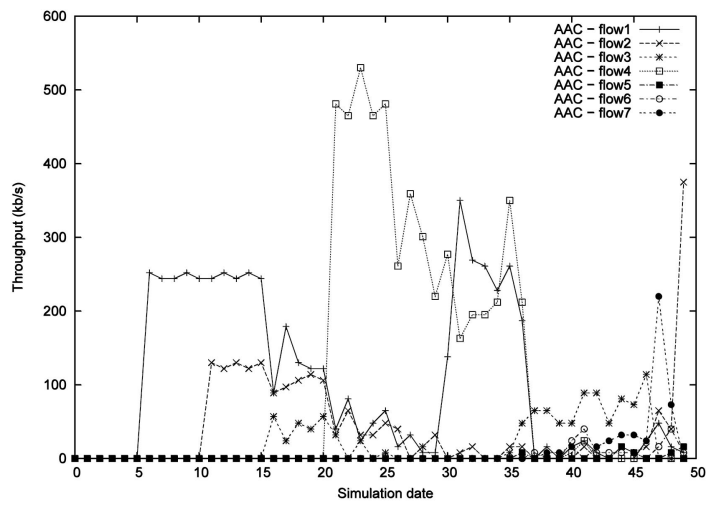

(b)

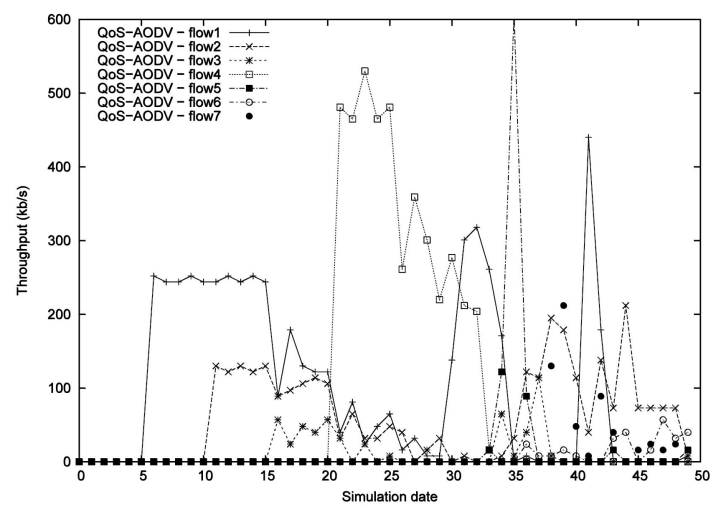

(c)

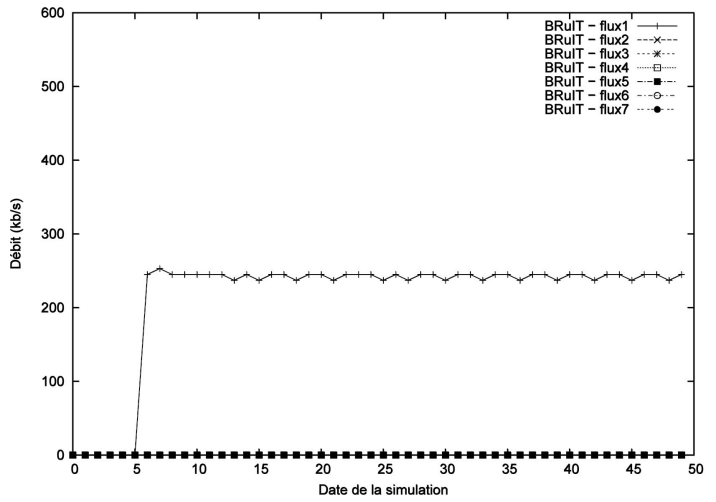

(d)

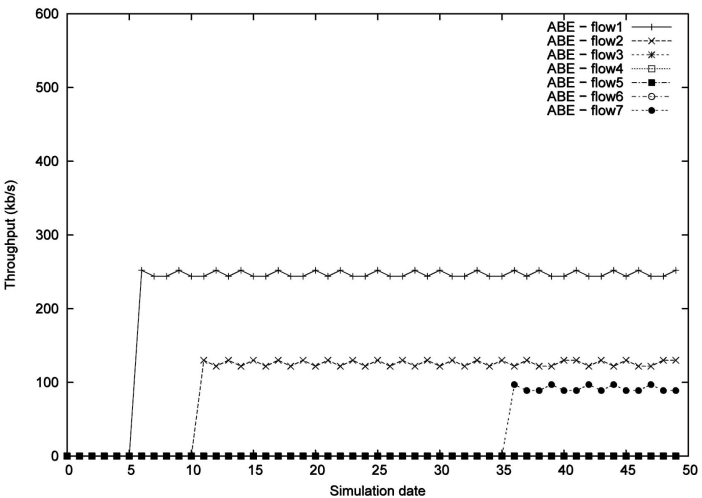

(e)

Fig. 10. Throughput of multihop flows with AODV, AAC, QoS-AODV, and ABE-AODV (2-Mbps medium capacity). (a) AODV. (b) AAC. (c) QoS-AODV. (d) BRulT. (e) ABE-AODV.

Hello packets and data flows increase, leading to an imprecise vision of the network real state.

ABE-AODV performs accurate evaluation in most situations because it does not rely solely on these broadcasted frames. When the network is not too loaded, the acceptance rate of QoS-AODV and AAC is smaller than BRuIT and ABE-AODV. QoS-AODV and AAC cause more false admissions due to the overestimation of the available bandwidth, and throughputs of close flows are degraded. However, when the network becomes loaded, BRuIT's acceptance rate decreases. Performing an underestimation of the available bandwidth, it tends to accept less flows than what the network is able to convey.

\subsection{Mobile Networks}

It is illusory to provide hard QoS guarantees when nodes are mobile. QoS violations appear due to the topology changes, which result either in route breakage or in unexpected variations of the available throughputs. In this situation, the route can either be finally broken or can be rebuilt at the source, or it may finally be rebuilt locally. Choosing between all these strategies is not in the scope of this paper. We present results on mobile networks only to give indication on the reactiveness of the raw bandwidth estimation approach, as protocols all exchange information at the same rate. 


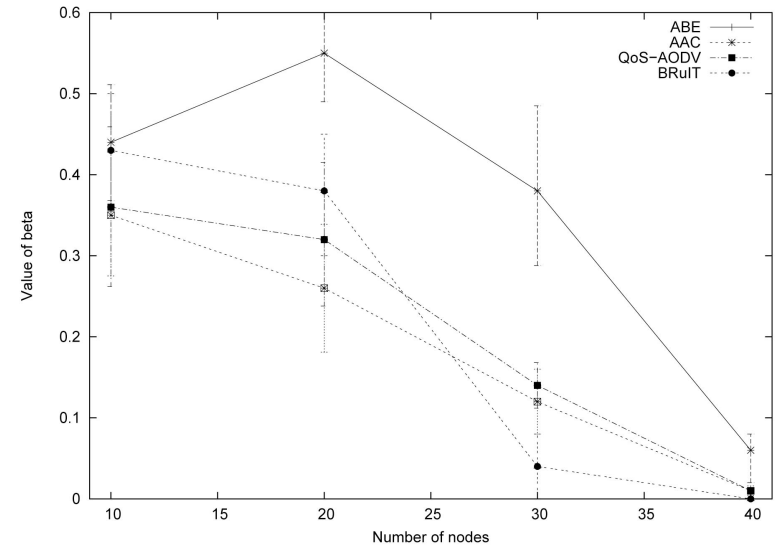

Fig. 11. Acceptance rate of flows with ABE-AODV, AAC, QoS-AODV, and BRulT.

To investigate the effect of mobility on flow throughput, we have performed simulations with 10 randomly positioned nodes. Five CBR traffics are generated with random throughputs and the starting dates of these flows are spaced by 2 seconds. Nodes move according to a random waypoint mobility model with a maximum speed of $20 \mathrm{~m} / \mathrm{s}$ and a pause time of 10 seconds. Each simulation lasts 100 seconds and the physical rate is of $2 \mathrm{Mbps}$.

In the scenario presented in this section, two routes are broken due to mobility after 40 seconds and a route appears at this date. Figs. $12 \mathrm{a}$ and $12 \mathrm{~b}$ show that after such break,

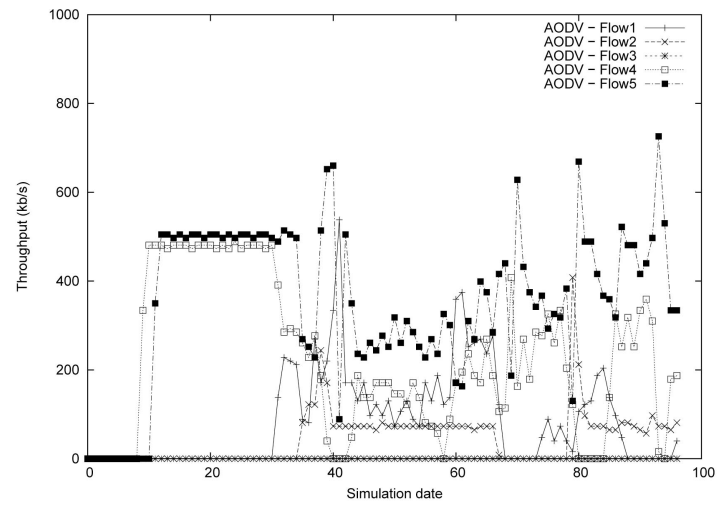

(a)

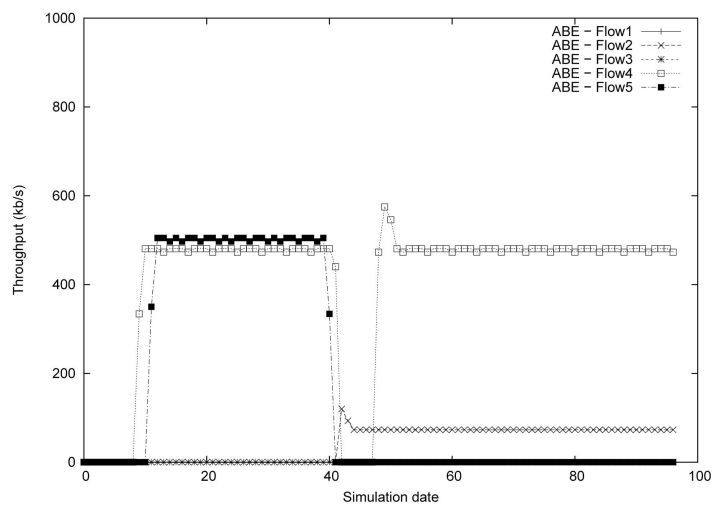

(c)
AAC fails to perform an accurate admission control, leading to unstable behavior. BRuIT (Fig. 12d) underestimates the medium capacity and fails to rebuild the routes when the requests are performed. Fig. 12c shows that ABE-AODV finds, after a certain delay, a new route for flow 4 and accepts flow 3 instead of flow 5 .

\section{Conclusions and Future Works}

In this paper, we have presented a new technique to compute the available bandwidth between two neighbor nodes and by extension along a path. This method combines channel monitoring to estimate each node's medium occupancy including distant emissions, probabilistic combination of these values to account for synchronization between nodes, estimation of the collision probability between each couple of nodes, and variable overhead's impact estimation. This mechanism only requires one-hop information communication and may be applied without generating a too high additional overhead.

This technique has been integrated in AODV for comparison purposes. We show the accuracy of the available bandwidth measurement through NS-2 simulations. These results show that single-hop flows and multihop flows are admitted more accurately, resulting in a better stability and overall performance. Results are encouraging in fixed networks as well as in mobile networks. From our point of view, these scenarios prove that the most

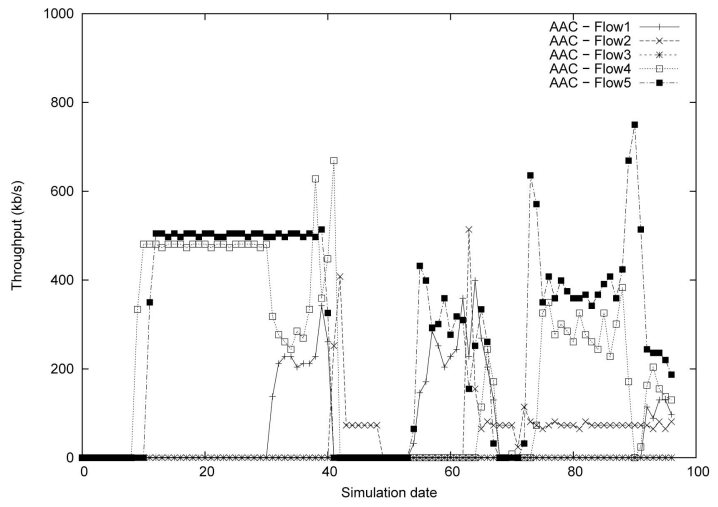

(b)

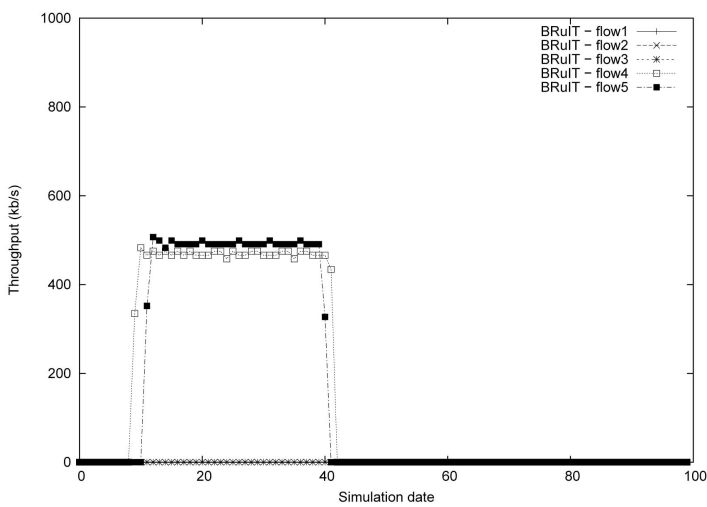

(d)

Fig. 12. Throughput obtained by AODV, AAC, BRulT, and ABE-AODV in mobile networks. (a) AODV. (b) AAC. (c) ABE-AODV. (d) BRulT. 
difficult point when designing a QoS protocol is not the routing process but the estimation of available resources through the network.

As future works, we plan to focus on two issues. First, in our current evaluation, we make no difference between the bandwidth consumed by QoS flows and the bandwidth consumed by best effort flows. Therefore, it may be possible that a node considers its available bandwidth on a link as almost null whereas the whole bandwidth is consumed by best effort flows. Decreasing the rate of these flows may lead to a higher acceptance rate of QoS flows. Differentiating flow types may also result in a better utilization of the network resources. In parallel, we are investigating the delay metric, as preliminary studies indicate that some parts of the approach described in this paper may be used or converted to this other important parameter.

\section{ACKNOWLEDGMENTS}

This work was partially supported by the European Project AEOLUS.

\section{REFERENCES}

[1] R. Prasad, M. Murray, C. Dovrolis, and K. Claffy, "Bandwidth Estimation: Metrics, Measurement Techniques, and Tools," IEEE Network, vol. 17, no. 6, pp. 27-35, Nov. 2003.

[2] M. Jain and C. Dovrolis, "End-to-End Available Bandwidth: Measurement Methodology, Dynamics, and Relation with TCP Throughput," IEEE/ACM Trans. Networking (TON '03), vol. 11, no. 4, pp. 537-549, Aug. 2003.

[3] B. Melander, M. Bjorkman, and P. Gunningberg, "A New Endto-End Probing Analysis Method for Estimating Bandwidth Bottlenecks," Proc. Fifth Global Internet Symp. (Global Internet) held in conjunction with Global Comm. Conf. (GLOBECOM '00), Nov. 2000.

[4] F.Y. Li, M. Haugea, A. Hafslund, O. Kure, and P. Spilling, "Estimating Residual Bandwidth in 802.11-Based Ad Hoc Networks: An Empirical Approach," Proc. Seventh Int'l Symp. Wireless Personal Multimedia Comm. (WPMC '04), Sept. 2004.

[5] A. Johnsson, B. Melander, and M. Björkman, "Bandwidth Measurement in Wireless Network," technical report, Mälardalen Univ., Mar. 2005.

[6] S.H. Shah, K. Chen, and K. Nahrstedt, "Dynamic Bandwidth Management for Single-Hop Ad Hoc Wireless Networks," Proc. First IEEE Int'l Conf. Pervasive Computing and Comm. (PerCom '03), Aug. 2003.

[7] K. Xu, K. Tang, R. Bagrodia, M. Gerla, and M. Bereschinsky, "Adaptive Bandwidth Management and QoS Provisioning in Large Scale Ad Hoc Networks," Proc. Military Comm. Conf. (MILCOM '03), Oct. 2003.

[8] R. de Renesse, M. Ghassemian, V. Friderikos, and A.H. Aghvami, "QoS Enabled Routing in Mobile Ad Hoc Networks," Proc. IEE Fifth Int'l Conf. 3G Mobile Comm. Technologies (IEE 3G), 2004.

[9] C. Chaudet and I.G. Lassous, "BRuIT-Bandwidth Reservation under InTerferences Influence," Proc. European Wireless (EW '02), Feb. 2002.

[10] C. Chaudet and I.G. Lassous, "Evaluation of the BRuIT Protocol," Proc. IEEE 61st Semiann. Vehicular Technology Conf. (VTC Spring '05), May 2005.

[11] Y. Yang and R. Kravets, "Contention Aware Admission Control for Ad Hoc Networks," IEEE Trans. Mobile Computing, vol. 4, pp. 363-377, 2005.

[12] K. Sanzgiri, I.D. Chakeres, and E.M. Belding-Royer, "Determining Intra-Flow Contention along Multihop Paths in Wireless Networks," Proc. First Int'l Conf. Broadband Networks (BROADNETS '04), Oct. 2004.

[13] R. de Renesse, M. Ghassemian, V. Friderikos, and A.H. Aghvami, "Adaptive Admission Control for Ad Hoc and Sensor Networks Providing Quality of Service," technical report, King College London, May 2005.
[14] V. Bharghavan, A.J. Demers, S. Shenker, and L. Zhang, "MACAW: A Media Access Protocol for Wireless LAN's," Proc. ACM SIGCOMM '94, pp. 212-225, 1994.

[15] C. Sarr, C. Chaudet, G. Chelius, and I.G. Lassous, "A Node-Based Available Bandwidth Evaluation in IEEE 802.11 Ad Hoc Networks," Int'l J. Parallel, Emergent and Distributed Systems, vol. 21, no. 6, 2006.

[16] G. Bianchi, "Performance Analysis of the IEEE 802.11 Distributed Coordination Function," J. Selected Areas in Comm., vol. 18, no. 3, pp. 535-547, Mar. 2000.

[17] L. Chen and W. Heinzelman, "QoS-Aware Routing Based on Bandwidth Estimation for Mobile Ad Hoc Networks," IEEE J. Selected Areas of Comm., vol. 3, 2005.

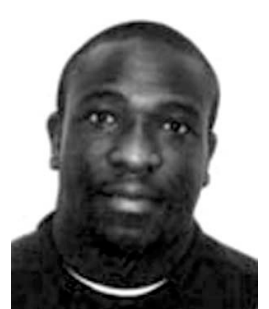

Cheikh Sarr received the $\mathrm{PhD}$ degree from the Institut National des Sciences Appliquées de Lyon, Villeurbanne, France, in 2007, under the supervision of Isabelle Guérin Lassous and Guillame Chelius. He is currently an associate professor in the Department of Computer Science, University of Thiès, Thiès, Senegal. His main research interest is quality of service in ad hoc networks, more specifically evaluation of different metrics like available bandwidth, end-to-end delay, and coexistence between quality of service and best effort traffic.

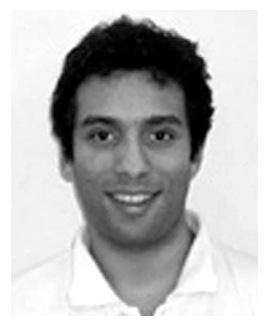

Claude Chaudet received the $\mathrm{PhD}$ degree in 2004 from the Institut National des Sciences Appliquées de Lyon, Villeurbanne, France, where his thesis was focused on QoS and bandwidth management in ad hoc networks. He is currently an associate professor at Telecom ParisTech (ENST). His research interests include wireless ad hoc and sensor networks, distributed algorithms, and resource sharing modeling.

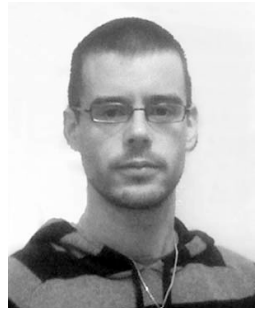

Guillaume Chelius received the PhD degree in 2004 from the Institut National des Sciences Appliquées de Lyon, Villeurbanne, France, where his thesis was focused on the architecture and communications in spontaneous multihop wireless networks. Since 2004, he is occupying a position of full researcher at the INRIA, Lyon, France. His research interests include communication layers and system aspects of sensor networks, distributed algorithms, and wireless

network simulation.

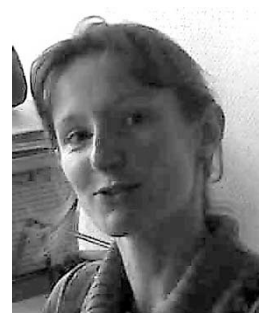

Isabelle Guérin Lassous has been a professor at the Université Claude Bernard Lyon 1, Villeurbanne, France and Ecole Normale Supérieure, Lyon, France, since September 2006. She was a full researcher at the Institut National de Recherche en Informatique et en Automatique, Le Chesnay, France. Her research area concerns wireless networks and distributed algorithms.

$\triangleright$ For more information on this or any other computing topic, please visit our Digital Library at www.computer.org/publications/dlib. 